quatrième série-tome $46 \quad$ fascicule $4 \quad$ juillet-aồt 2013

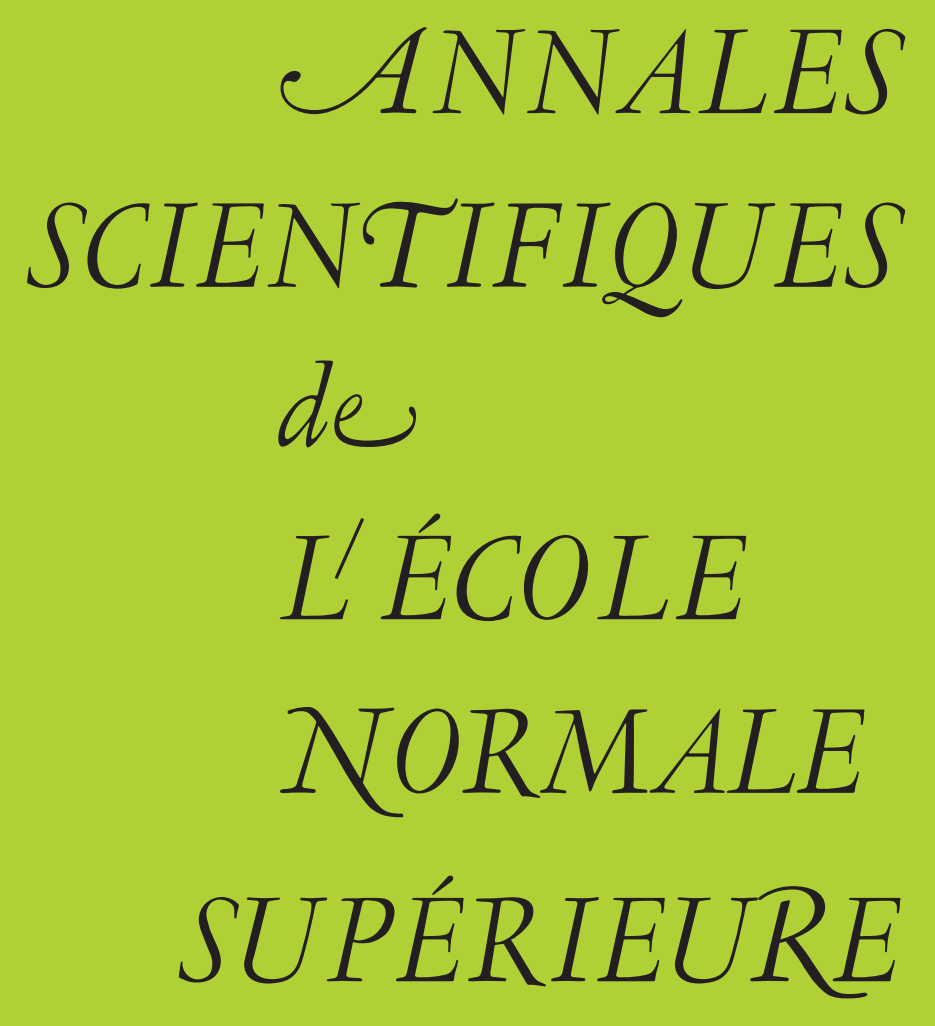

Jun-Muk HWANG

Varieties of minimal rational tangents of codimension 1 
Ann. Scient. Éc. Norm. Sup.

$4^{\text {e }}$ série, t. 46, 2013, p. 629 à 649

\title{
VARIETIES OF MINIMAL RATIONAL TANGENTS OF CODIMENSION 1
}

\author{
BY JUN-MUK HWANG
}

\begin{abstract}
Let $X$ be a uniruled projective manifold and let $x$ be a general point. The main result of [2] says that if the $\left(-K_{X}\right)$-degrees (i.e., the degrees with respect to the anti-canonical bundle of $X$ ) of all rational curves through $x$ are at least $\operatorname{dim} X+1$, then $X$ is a projective space. In this paper, we study the structure of $X$ when the $\left(-K_{X}\right)$-degrees of all rational curves through $x$ are at least $\operatorname{dim} X$.

Our study uses the projective variety $\mathscr{C}_{x} \subset \mathbb{P} T_{x}(X)$, called the VMRT at $x$, defined as the union of tangent directions to the rational curves through $x$ with minimal $\left(-K_{X}\right)$-degree. When the minimal $\left(-K_{X}\right)$-degree of rational curves through $x$ is equal to $\operatorname{dim} X$, the VMRT $\mathscr{C}_{x}$ is a hypersurface in $\mathbb{P} T_{x}(X)$. Our main result says that if the VMRT at a general point of a uniruled projective manifold $X$ of dimension $\geq 4$ is a smooth hypersurface, then $X$ is birational to the quotient of an explicit rational variety by a finite group action. As an application, we show that, if furthermore $X$ has Picard number 1 , then $X$ is biregular to a hyperquadric.
\end{abstract}

RÉsumÉ. - Soit $X$ une variété projective uniréglée et soit $x$ un point général. D'après le résultat principal de [2], si le degré par rapport à $-K_{X}$ de toute courbe rationnelle passant par $x$ est au moins égal à $\operatorname{dim}(X)+1$, alors $X$ est un espace projectif. Dans cet article, nous étudions la structure de $X$ sous l'hypothèse que le degré par rapport à $-K_{X}$ de toute courbe rationnelle passant par $x$ est au moins égal à $\operatorname{dim}(X)$.

Notre étude repose sur la variété projective $\mathscr{C}_{x} \subset \mathbb{P} T_{x}(X)$ que nous appelons la VMRT (variété des tangentes des courbes rationnelles minimales) en $x$ et qui est définie comme la réunion de toutes les directions tangentes aux courbes rationnelles passant par $x$ dont le degré par rapport à $-K_{X}$ est minimal. Lorsque ce degré est égal à $\operatorname{dim}(X)$, la VMRT $\mathscr{C}_{x}$ est une hypersurface de $\mathbb{P} T_{x}(X)$. Notre résultat principal affirme que si la VMRT en un point général d'une variété projective uniréglée $X$ de dimension $\geq 4$ est une hypersurface, alors $X$ est birationnelle au quotient d'une variété rationnelle explicite par l'action d'un groupe fini. Si, de plus, le rang du groupe de Picard de $X$ est égal à 1 , nous en déduisons que $X$ est une hypersurface quadrique d'un espace projectif.

Supported by National Researcher Program 2010-0020413 of NRF and MEST. 


\section{Introduction and statement of main results}

We will work over the complex numbers. For a projective manifold $X$, we have the notion of the (normalized) space RatCurves ${ }^{\mathrm{n}}(X)$ of rational curves on $X$ (cf. [11] Section II.2 for the definition). When $X$ is uniruled, this space $\operatorname{RatCurves}^{\mathrm{n}}(X)$ has an irreducible component $\mathcal{K}$ such that the subscheme $\mathcal{K}_{x}$ consisting of members of $\mathcal{K}$ through a general point $x \in X$ is non-empty. If furthermore $\mathcal{K}_{x}$ is projective, the component $\mathcal{K}$ is called a family of minimal rational curves and its members are called minimal rational curves of $X$. For a uniruled projective manifold $X$, a family of minimal rational curves always exists. For example, fix an ample line bundle $L$ on $X$ and pick a rational curve $C$ through a general point of $X$ which has minimal degree with respect to $L$. Then the component of $\operatorname{RatCurves}^{\mathrm{n}}(X)$ containing $C$ is a family of minimal rational curves. Minimal rational curves play a crucial role in the geometry of $X$ and have been much studied for that reason.

Given a family $\mathcal{K}$ of minimal rational curves, denote by $\operatorname{deg}(\mathcal{K})$ the degree of members of $\mathcal{K}$ with respect to the anti-canonical divisor $-K_{X}$. Then it is well-known that $\operatorname{deg}(\mathcal{K}) \leq \operatorname{dim} X+1$ (e.g., [11] Corollary IV.1.15). When $\operatorname{deg}(\mathcal{K})$ is maximal, i.e., $\operatorname{deg}(\mathcal{K})=\operatorname{dim} X+1$, the biregular structure of $X$ is completely known: Theorem 0.2 of [2] says that $X$ is biregular to projective space and minimal rational curves are lines.

In this paper, we study the next-to-maximal case, i.e., when $\operatorname{deg}(\mathcal{K})=\operatorname{dim} X$. To see what is to be expected, recall the following examples which are introduced in Example 1.7 of [5].

ExAmple 1.1. - Let $Z \subset \mathbb{P}^{n-1}, n \geq 3$, be a submanifold. Regard $Z$ as a submanifold of a hyperplane $\mathbb{P}^{n-1} \subset \mathbb{P}^{n}$. Let $\beta: X_{Z} \rightarrow \mathbb{P}^{n}$ be the blow-up of $\mathbb{P}^{n}$ along $Z$. Let $\mathcal{K}_{Z}$ be the component of RatCurves ${ }^{\mathrm{n}}\left(X_{Z}\right)$ determined by the proper transforms of lines on $\mathbb{P}^{n}$ intersecting $Z$. Then $\mathcal{K}_{Z}$ is a family of minimal rational curves on $X_{Z}$. If $Z \subset \mathbb{P}^{n-1}$ is a hypersurface, then $\operatorname{deg}\left(\mathcal{K}_{Z}\right)=n$.

Another example can be obtained by taking a cyclic quotient of Example 1.1:

ExAmple 1.2. - In the setting of Example 1.1, let $G$ be a finite group acting on $\mathbb{P}^{n}$ preserving the two submanifolds $Z \subset \mathbb{P}^{n-1} \subset \mathbb{P}^{n}$. The $G$-action can be lifted to a $G$-action on $X_{Z}$ and the quotient $X_{Z} / G$ exists as a normal variety. Let $\overline{X_{Z} / G}$ be a desingularization of $X_{Z} / G$ which leaves the smooth locus intact. For some choice of $Z$ and $G$, the family of rational curves given by the proper images of members of $\mathcal{K}_{Z}$ in $\overline{X_{Z} / G}$ becomes a family of minimal rational curves on $\overline{X_{Z} / G}$. For example, when $Z$ is a Fermat hypersurface of large degree, we can find a cyclic group $G$ acting on $\mathbb{P}^{n}$ preserving $Z \subset \mathbb{P}^{n-1}$ such that the action is free outside a finite set $F \subset \mathbb{P}^{n}$ with $F \cap Z=\varnothing$. When we regard $F$ as a subset in $X_{Z}$, the $G$-action on $X_{Z}$ is free outside $F \subset X_{Z}$ which is disjoint from members of $\mathcal{K}_{Z}$ through a general point. Thus the quotient $X_{Z} \rightarrow X_{Z} / G$ is unramified at all points of the members of $\mathcal{K}_{Z}$ through general points, which shows that $\mathcal{K}_{Z}$ descends to a family of minimal rational curves on $\widehat{X_{Z} / G}$.

More examples can be constructed by suitable birational transformations of $X_{Z}$ as follows.

4 e SÉRIE - TOME $46-2013-$ No $^{\circ}$ 
EXAmple 1.3. - Given a projective manifold $X$ and a family of minimal rational curves $\mathcal{K}$ with $\operatorname{deg}(\mathcal{K})=\operatorname{dim} X$, there exists a non-trivial birational transformation $\Phi: X \rightarrow X^{\prime}$ such that the proper images of members of $\mathcal{K}$ give rise to a family of minimal rational curves $\mathcal{K}^{\prime}$ on $X^{\prime}$. Then $\operatorname{deg}\left(\mathcal{K}^{\prime}\right)=\operatorname{dim} X^{\prime}$. For example, for any finite subset $F \subset X$, all members of $\mathcal{K}$ through a general point $x \in X$ are disjoint from $F$. Thus the blow-up $\Phi: X \rightarrow \mathrm{Bl}_{F}(X)$ provides an example of such birational transformations.

These examples show that, unlike in the case of $\operatorname{deg}(\mathcal{K})=\operatorname{dim} X+1$, there is no simple biregular classification of $X$ in the case of $\operatorname{deg}(\mathcal{K})=\operatorname{dim} X$. What we can hope is to give a birational classification of $X$ together with a description of $\mathcal{K}$. We achieve this for projective manifolds of dimension $\geq 4$, modulo one technical assumption. To explain this technical assumption, let us recall the notion of the variety of minimal rational tangents (to be abbreviated as VMRT) associated to a family of minimal rational curves $\mathcal{K}$. The $V M R T$ at a general point $x \in X$ is the subvariety $\mathscr{C}_{x} \subset \mathbb{P} T_{x}(X)$ defined as the set of tangent directions at $x$ of the members of $\mathcal{K}_{x}$ (cf. [4] Section 1.3). The dimension of $\mathscr{C}_{x}$ is equal to $\operatorname{deg}(\mathcal{K})-2$. The technical assumption we need is the following.

$\left.{ }^{*}\right)$ The VMRT $\mathscr{C}_{x}$ at a general point $x \in X$ is smooth.

It has been conjectured that $(*)$ is true for any $X$ and any $\mathcal{K}$. The main result of [8] says that the normalization of $\mathscr{C}_{x}$ is smooth, so $\left(^{*}\right)$ is equivalent to the normality of $\mathscr{C}_{x}$. In specific problems involving minimal rational curves, the assumption $(*)$ is often verifiable directly. For example, when $\operatorname{deg}(\mathcal{K})=\operatorname{dim} X+1, \mathscr{C}_{x}=\mathbb{P} T_{x}(X)$ is trivially smooth, which plays an implicit role in [2]. It is also easy to check (*) in Examples 1.1 and 1.2. In fact, $\mathscr{C}_{x} \subset \mathbb{P} T_{x}\left(X_{Z}\right)$ is isomorphic to $Z \subset \mathbb{P}^{n-1}$ as projective subvariety. In this sense, it is reasonable to keep (*) as a working hypothesis when studying minimal rational curves.

Our main result is the following. Note that under the assumption of $\left(^{*}\right)$ and $\operatorname{deg}(\mathcal{K})=n$, the VMRT $\mathscr{C}_{x}$ is a smooth hypersurface in $\mathbb{P} T_{x}(X)$.

TheOrem 1.4. - Let $X$ be a uniruled projective manifold of dimension $n \geq 4$ with a family $\mathcal{K}$ of minimal rational curves such that the $V M R T \mathscr{C}_{x} \subset \mathbb{P} T_{x}(X)$ at a general point $x \in X$ is a smooth hypersurface of degree $m \geq 3$. Then there exist a smooth hypersurface $Z \subset \mathbb{P}^{n-1}$ of degree $m$, a finite group $G$ acting on $\mathbb{P}^{n}$ preserving $Z \subset \mathbb{P}^{n-1}$ and a birational map $\Phi: X_{Z} / G \rightarrow X$ such that the dominant rational map $\Psi: X_{Z} \rightarrow X$ defined by the composition of $\Phi$ and the quotient $X_{Z} \rightarrow X_{Z} / G$ sends general members of $\mathcal{K}_{Z}$ to those of $\mathcal{K}$.

The assumption $m \geq 3$ in Theorem 1.4 is harmless. When the degree $m$ of the hypersurface $\mathscr{C}_{x}$ is 1 or 2, the structure of $X$ is already well-understood. When $m=1$, Theorem 1.1 of [1] says that there exist a Zariski open subset $X^{o} \subset X$ and a $\mathbb{P}^{n-1}$-fibration $X^{o} \rightarrow B$ to a (quasi-projective) curve $B$ such that members of $\mathcal{K}$ correspond to lines in the $\mathbb{P}^{n-1}$-fibers. When $m=2$, i.e., $\mathscr{C}_{x}$ is a hyperquadric, the conclusion of Theorem 1.4 follows from the work of Mok ([13]) as a variation of its Main Theorem. Strictly speaking, [13] is written under the assumption that $X$ has Picard number 1 and its Main Theorem is stated with that assumption. However, those arguments in [13] that are independent from that assumption and our argument in Section 5 provide the proof we need. 
Theorem 1.4 gives a reasonably satisfactory classification under the technical assumption (*). However, to be a complete classification, we need to classify the pairs $(Z, G)$ which actually occur and determine the birational type of $X_{Z} / G$. This seems to require a substantial work of quite different nature from the main theme of the current paper. Thus we will not touch upon these questions in the current paper and leave them for future research of interested readers.

Our approach to Theorem 1.4 is based on the study of geometric structures arising from minimal rational curves. To fix the terminology, we give the definition here.

Definition 1.5. - On a uniruled projective manifold $X$ with a family $\mathcal{K}$ of minimal rational curves, the $V M R T$-structure $\mathscr{C} \subset \mathbb{P} T(X)$ is the irreducible subvariety defined by the closure of the union of the VMRT's $\mathscr{C}_{x} \subset \mathbb{P} T_{x}(X)$ as $x$ varies over general points of $X$.

In contrast to previous terminology in [4], [8], etc., the term 'structure' is used here to emphasize our view-point that this subvariety should be viewed as a differential geometric object. In fact, a key notion is the equivalence of VMRT-structures in the following sense.

Definition 1.6. - Given two uniruled projective manifolds $X^{1}$ and $X^{2}$ with families of minimal rational curves $\mathcal{K}^{1}$ and $\mathcal{K}^{2}$ respectively, the corresponding VMRT-structure $\mathscr{C}^{1} \subset \mathbb{P} T\left(X^{1}\right)$ at a point $x^{1} \in X^{1}$ is equivalent to the VMRT-structure $\mathscr{C}^{2} \subset \mathbb{P} T\left(X^{2}\right)$ at $x^{2} \in X^{2}$, if there exist an analytic neighborhood $x^{1} \subset U^{1} \subset X^{1}$ (resp. $x^{2} \subset U^{2} \subset X^{2}$ ) and a biholomorphic map $\psi: U^{1} \rightarrow U^{2}$ whose differential $d \psi: \mathbb{P} T\left(U^{1}\right) \rightarrow \mathbb{P} T\left(U^{2}\right)$ sends $\mathscr{C}^{1} \cap \mathbb{P} T\left(U^{1}\right)$ to $\mathscr{C}^{2} \cap \mathbb{P} T\left(U^{2}\right)$ biholomorphically.

To see the relevance of the equivalence of VMRT-structures in Theorem 1.4, recall the following result.

Theorem 1.7 (Main Theorem in [7]). - Let $X^{1}$ (resp. $X^{2}$ ) be a uniruled projective manifold of Picard number 1, not biregular to projective space. Assume that $X^{1}$ (resp. $X^{2}$ ) has a family of minimal rational curves $\mathcal{K}^{1}$ (resp. $\mathcal{K}^{2}$ ) such that the VMRT $\mathscr{C}_{x_{1}}^{1} \subset \mathbb{P} T_{x_{1}}\left(X^{1}\right)$ at a general point $x^{1} \in X^{1}$ (resp. $x^{2} \in X^{2}$ ) is smooth and irreducible, and the VMRT-structure at $x^{1}$ is equivalent to the VMRT-structure at $x^{2}$ in the sense of Definition 1.6. Then the local equivalence map $\psi$ in Definition 1.6 extends to a biregular morphism $\Psi: X^{1} \rightarrow X^{2}$ which sends members of $\mathcal{K}^{1}$ to members of $\mathcal{K}^{2}$.

It turns out that we can use some analogue of Theorem 1.7 to reduce the proof of Theorem 1.4, to showing that the VMRT-structure of $X$ at a general point is equivalent to that of $X_{Z}$ in Example 1.1. The argument we need for this reduction is a variation of the proof of Theorem 1.7 in [7]. This will be explained in Section 5.

From this, we may say that the gist of the matter is to study the equivalence problem for the VMRT-structure in the setting of Theorem 1.4. In [5], this equivalence problem is studied and the following result is proved (Theorem 1.11 in [5]).

THeOREM 1.8. - Let $X$ be a uniruled projective manifold of dimension $n \geq 3$ with a family $\mathcal{K}$ of minimal rational curves with $\operatorname{deg}(\mathcal{K})=n$. Assume that for a general $x \in X$,

(i) the VMRT $\mathscr{C}_{x} \subset \mathbb{P} T_{x}(X)$ is smooth;

(ii) the hypersurface $\mathscr{C}_{x} \subset \mathbb{P} T_{x}(X)$ has degree $m \geq 4$; and

$4^{\mathrm{e}}$ SÉRIE - TOME $46-2013-\mathrm{N}^{\circ} 4$ 
(iii) $\mathscr{C}$ is isotrivial, namely, for any $y \in X$ in a neighborhood of $x$, the hypersurface $\mathscr{C}_{y} \subset \mathbb{P} T_{y}(X)$ is projectively equivalent to a fixed hypersurface $Z \subset \mathbb{P}^{n-1}$.

Then the VMRT-structure $\mathscr{C}$ is locally flat at a general point (cf. Definition 3.14), or equivalently, equivalent to the VMRT-structure at a general point of $X_{Z}$ (cf. Example 4.2).

Among the three assumptions in Theorem 1.8, (i) is exactly our technical assumption $(*)$, and does not damage the generality of the statement. However the assumptions (ii) and (iii) are restrictive. It is unnatural to exclude $m=3$ in (ii). The isotriviality assumption in (iii) is, by far, the strongest condition in Theorem 1.8. In [5], (iii) is imposed to employ the differential geometric machinery developed by É. Cartan. From the view-point of algebraic geometry, this assumption is most unsatisfactory.

The essence of the present work is to remove the two assumptions (ii) and (iii). More precisely, we prove the following, which is the essential result of this paper.

THeOREM 1.9. - Let $X$ be a uniruled projective manifold of dimension $n \geq 4$ with a family $\mathcal{K}$ of minimal rational curves with $\operatorname{deg}(\mathcal{K})=n$. Assume that for a general $x \in X$, the hypersurface $\mathscr{C}_{x} \subset \mathbb{P} T_{x}(X)$ is smooth of degree $m \geq 3$. Then the VMRT-structure is locally flat at a general point.

Note that Theorem 1.9, as well as Theorem 1.4, is stated only for $n \geq 4$, while Theorem 1.8 holds for $n \geq 3$. I have no idea whether an analogue of Theorem 1.9 is true when $n=3$. We need $n \geq 4$ in Theorem 1.9 because it is proved via the following more general result. For the definition of the terms and the notation here, see Section 2.

TheOREM 1.10. - Let $\mathscr{C} \subset \mathbb{P} T(X)$ be a VMRT-structure on a uniruled projective manifold. Assume that for a general $x \in X$, the VMRT $\mathscr{C}_{x}$ is smooth and irreducible. Assume furthermore that

(1) $\mathscr{C}_{x}$ is linearly normal;

(2) $\mathscr{C}_{x}$ is tangentially non-degenerate;

(3) $H^{0}\left(\mathscr{C}_{x}, T\left(\mathscr{C}_{x}\right)\right)=0$;

(4) $H^{1}\left(\mathscr{C}_{x}, \mathscr{\vartheta}_{\mathscr{C}_{x}}\right)=0$; and

(5) $\operatorname{dim} H^{1}\left(\mathscr{C}_{x},\left.T\left(\mathscr{C}_{x}\right) \otimes N_{\mathscr{C}_{x}}^{*} \otimes \mathscr{\theta}(1)\right|_{\mathscr{C}_{x}}\right) \leq \operatorname{dim} H^{0}\left(\mathscr{C}_{x},\left.\Theta(1)\right|_{\mathscr{C}_{x}}\right)$.

Then the VMRT-structure is locally flat at a general point.

It is easy to check that the conditions (1)-(5) hold for smooth hypersurfaces in $\mathbb{P}^{n-1}$ of degree $\geq 3$ if $n \geq 4$ as explained in Proposition 2.4 below. Thus Theorem 1.10 implies Theorem 1.9. But the condition (5) is not true if $n=3$ (cf. Remark 2.5), which is why we need the assumption $n \geq 4$ in Theorem 1.9.

Theorem 1.10 is proved by showing that a certain exact sequence of vector bundles on $\mathscr{C}$ over an open subset of $X$ splits (cf. Proposition 4.5). This splitting is obtained from the deformation theory of minimal rational curves and implies the vanishing of the KodairaSpencer class for $\mathscr{C}_{x}$ 's which leads to the isotriviality.

We expect that Theorem 1.4 and Theorem 1.9 will be useful in the study of uniruled projective manifolds. As an application, we will prove 
Theorem 1.11. - Let $X$ be a Fano manifold of Picard number 1 of dimension $\geq 3$ such that the VMRT $\mathscr{C}_{x} \subset \mathbb{P} T_{x}(X)$ at a general point $x \in X$ associated to a family of minimal rational curves is a smooth hypersurface. Then $X$ is biregular to the hyperquadric.

Theorem 1.11 is a special case of Miyaoka's result in [12]. Miyaoka's result is stronger because it does not require the assumption $(*)$. However, our proof of Theorem 1.11 via Theorem 1.9, to be explained in Section 6, is completely different from the proof in [12]. The method of [12] is based on the study of certain rational curves of higher degree and is quite technical. Since it depends heavily on the assumption $\operatorname{Pic}(X) \cong \mathbb{Z}$, the approach of [12] looks hard to yield results like Theorem 1.4.

One may ask whether we can go further to the cases of $\operatorname{deg}(\mathcal{K})<\operatorname{dim} X$. A general classification result like Theorem 1.4 is hopeless even for $\operatorname{deg}(\mathcal{K})=\operatorname{dim} X-1$. However, since Theorem 1.10 does not require $\mathscr{C}_{x}$ is of codimension 1 , it may be applicable in some cases. It looks plausible that there are examples of projective submanifolds satisfying the conditions (1)-(5) of Theorem 1.10 other than hypersurfaces. In this regard, it is worth noticing that the conditions (1)-(4) in Theorem 1.10 hold for smooth complete intersections of small codimension. But the condition (5) does not hold even for a smooth complete intersection of codimension 2. As an example, when $X \subset \mathbb{P}^{n+1}$ is an $n$-dimensional cubic hypersurface, the VMRT $\mathscr{C}_{x} \subset \mathbb{P} T_{x}(X)$ at a general point $x \in X$ is the complete intersection of a quadric and a cubic. Thus $\mathscr{C}_{x}$ satisfies (1)-(4) if $n$ is sufficiently large, but does not satisfy (5). In fact, the VMRT-structure is not locally flat, because if it is locally flat, $X$ must be quasihomogeneous by Proposition 6.2 below, which is absurd because the automorphism group of a smooth cubic hypersurface is finite.

To close the introduction, let us remark that there are some previous results, e.g., [3] and [13], where the knowledge of the projective geometry of $\mathscr{C}_{x}$ at a general point $x \in X$ implies the local flatness of the VMRT-structure. However, in all these previous results, $\mathscr{C}_{x} \subset \mathbb{P} T_{x}(X)$ is rigid, i.e., the structure is a priori isotrivial. Our Theorem 1.9 is the first result where the local flatness holds even though the projective variety $\mathscr{C}_{x}$ can have nontrivial deformation.

\section{Some results on subvarieties of projective space}

Notation 2.1. - Given a vector space $V$, its projectivization $\mathbb{P} V$ is the variety of 1-dimensional subspaces of $V$. For a non-singular projective variety $Z \subset \mathbb{P} V$, we denote by $N_{Z}$ the normal bundle $\left.T(\mathbb{P} V)\right|_{Z} / T(Z)$. We have the conormal bundle sequence

$$
\left.0 \rightarrow N_{Z}^{*} \rightarrow T^{*}(\mathbb{P} V)\right|_{Z} \rightarrow T^{*}(Z) \rightarrow 0 .
$$

Proposition 2.2. - For a non-singular projective variety $Z \subset \mathbb{P V}$, denote by $L:=\left.\Theta(1)\right|_{Z}$, the restriction of the hyperplane line bundle. The conormal bundle sequence induces an exact sequence

$$
\begin{gathered}
H^{0}\left(Z,\left.T(Z) \otimes T^{*}(\mathbb{P} V)\right|_{Z} \otimes L\right) \rightarrow H^{0}\left(Z, T(Z) \otimes T^{*}(Z) \otimes L\right) \stackrel{\chi}{\rightarrow} \\
H^{1}\left(Z, T(Z) \otimes N_{Z}^{*} \otimes L\right) \rightarrow H^{1}\left(Z,\left.T(Z) \otimes T^{*}(\mathbb{P} V)\right|_{Z} \otimes L\right) .
\end{gathered}
$$

4 e SÉRIE - TOME $46-2013-$ NNo $^{\circ}$ 
The homomorphism $\chi$ is bijective if $H^{0}(Z, T(Z))=0$ and

$$
\operatorname{dim} H^{1}\left(Z, T(Z) \otimes N_{Z}^{*} \otimes L\right) \leq \operatorname{dim} H^{0}(Z, L) .
$$

Furthermore, in this case, $H^{0}\left(Z, \operatorname{End}^{0}(T(Z)) \otimes L\right)=0$, where $\operatorname{End}^{0}(\cdot)$ denotes the space of traceless endomorphisms.

Proof. - The exact sequence results from the conormal bundle sequence in Notation 2.1 by tensor product with $T(Z) \otimes L$.

Denote by $\widetilde{V}$ the trivial vector bundle on $Z$ with fiber $V$. From the Euler sequence

$$
\left.0 \rightarrow L^{*} \rightarrow \widetilde{V} \rightarrow T(\mathbb{P} V)\right|_{Z} \otimes L^{*} \rightarrow 0
$$

we have

$$
H^{0}\left(Z,\left.T(Z) \otimes T^{*}(\mathbb{P} V)\right|_{Z} \otimes L\right) \subset H^{0}\left(Z, T(Z) \otimes \widetilde{V}^{*}\right)=V^{*} \otimes H^{0}(Z, T(Z))=0 .
$$

Thus the homomorphism $\chi$ is injective. From

$$
H^{0}\left(Z, T(Z) \otimes T^{*}(Z) \otimes L\right)=H^{0}\left(Z, \operatorname{End}^{0}(T(Z)) \otimes L\right) \oplus H^{0}(Z, L),
$$

the inequality

$$
\operatorname{dim} H^{1}\left(Z, T(Z) \otimes N_{Z}^{*} \otimes L\right) \leq \operatorname{dim} H^{0}(Z, L)
$$

implies $H^{0}\left(Z, \operatorname{End}^{0}(T(Z)) \otimes L\right)=0$ and the bijectivity of $\chi$.

Definition 2.3. - A projective variety $Z \subset \mathbb{P} V$ is linearly normal if the restriction homomorphism

$$
H^{0}(\mathbb{P} V, \theta(1)) \longrightarrow H^{0}(Z, L)
$$

is bijective. For a non-singular projective variety $Z \subset \mathbb{P} V$, its variety of tangent lines is the subvariety in $\mathbb{P}\left(\wedge^{2} V\right)$ defined as the closure of the union of the points in $\mathbb{P}\left(\wedge^{2} V\right)$ corresponding to lines of $\mathbb{P} V$ tangent to $Z$. We say that $Z$ is tangentially non-degenerate if its variety of tangent lines is non-degenerate in $\mathbb{P}\left(\wedge^{2} V\right)$, i.e., not contained in any hyperplane in $\mathbb{P}\left(\wedge^{2} V\right)$.

Proposition 2.4. - Let $Z \subset \mathbb{P}^{n-1}$ be a smooth hypersurface of degree $m \geq 3$ with $n \geq 4$. Then

(1) $Z$ is linearly normal;

(2) $Z$ is tangentially non-degenerate;

(3) $H^{0}(Z, T(Z))=0$;

(4) $H^{1}\left(Z, \theta_{Z}\right)=0$; and

(5) $\operatorname{dim} H^{1}\left(Z, T(Z) \otimes N_{Z}^{*} \otimes L\right)=n$.

Proof. - The conditions (1)-(4) are well-known for smooth hypersurfaces of degree $m \geq 3$ with $n \geq 4$. Let us check (5). Since $N_{Z}^{*} \cong L^{-m}$, we need to check

$$
\operatorname{dim} H^{1}\left(Z, T(Z) \otimes L^{1-m}\right)=n .
$$

From the sequence

$$
\left.0 \longrightarrow T(Z) \longrightarrow T\left(\mathbb{P}^{n-1}\right)\right|_{Z} \longrightarrow L^{m} \longrightarrow 0
$$

we have

$H^{0}\left(Z,\left.T\left(\mathbb{P}^{n-1}\right)\right|_{Z} \otimes L^{1-m}\right) \rightarrow H^{0}(Z, L) \rightarrow H^{1}\left(Z, T(Z) \otimes L^{1-m}\right) \rightarrow H^{1}\left(Z,\left.T\left(\mathbb{P}^{n-1}\right)\right|_{Z} \otimes L^{1-m}\right)$. 
From $\left.T\left(\mathbb{P}^{n-1}\right)\right|_{Z} \otimes L^{1-m} \cong \Omega_{\mathbb{P}^{n-1}}^{n-2}(n-m+1)$, it suffices to prove

$$
H^{0}\left(Z,\left.\Omega_{\mathbb{P}^{n-1}}^{n-2}(n-m+1)\right|_{Z}\right)=H^{1}\left(Z,\left.\Omega_{\mathbb{P}^{n-1}}^{n-2}(n-m+1)\right|_{Z}\right)=0 .
$$

From the sequence on $\mathbb{P}^{n-1}$,

$$
\left.0 \longrightarrow \Omega_{\mathbb{P}^{n-1}}^{n-2}(n-2 m+1) \longrightarrow \Omega_{\mathbb{P}^{n-1}}^{n-2}(n-m+1) \longrightarrow \Omega_{\mathbb{P}^{n-1}}^{n-2}(n-m+1)\right|_{Z} \longrightarrow 0,
$$

we have

$H^{0}\left(\mathbb{P}^{n-1}, \Omega_{\mathbb{P}^{n-1}}^{n-2}(n-m+1)\right) \rightarrow H^{0}\left(Z,\left.\Omega_{\mathbb{P}^{n-1}}^{n-2}(n-m+1)\right|_{Z}\right) \rightarrow H^{1}\left(\mathbb{P}^{n-1}, \Omega_{\mathbb{P}^{n-1}}^{n-2}(n-2 m+1)\right)$ and

$H^{1}\left(\mathbb{P}^{n-1}, \Omega_{\mathbb{P}^{n-1}}^{n-2}(n-m+1)\right) \rightarrow H^{1}\left(Z,\left.\Omega_{\mathbb{P}^{n-1}}^{n-2}(n-m+1)\right|_{Z}\right) \rightarrow H^{2}\left(\mathbb{P}^{n-1}, \Omega_{\mathbb{P}^{n-1}}^{n-2}(n-2 m+1)\right)$.

Thus the desired vanishing follows from the well-known vanishing (e.g., p.8 of [14]) of

$$
\begin{aligned}
& H^{0}\left(\mathbb{P}^{n-1}, \Omega_{\mathbb{P}^{n-1}}^{n-2}(n-m+1)\right), H^{1}\left(\mathbb{P}^{n-1}, \Omega_{\mathbb{P}^{n-1}}^{n-2}(n-2 m+1)\right), \\
& H^{1}\left(\mathbb{P}^{n-1}, \Omega_{\mathbb{P}^{n-1}}^{n-2}(n-m+1)\right), H^{2}\left(\mathbb{P}^{n-1}, \Omega_{\mathbb{P}^{n-1}}^{n-2}(n-2 m+1)\right)
\end{aligned}
$$

for $n \geq 4$ and $m \geq 3$.

RemARK 2.5. - When $n=3$, i.e., $Z$ is a plane curve $Z \subset \mathbb{P}^{2}$,

$$
\operatorname{dim} H^{1}\left(Z, T(Z) \otimes N_{Z}^{*} \otimes L\right)=\operatorname{dim} H^{0}\left(Z, K_{Z}^{2} \otimes L^{m-1}\right)=\frac{m(5 m-11)}{2}>3 .
$$

Thus the statement (5) does not hold when $n=3$.

\section{Cone structures with $\mathscr{P}$-splitting connections}

Notation 3.1. - Given a projective submanifold $Z \subset \mathbb{P} V$, the homogenous cone of $Z$ will be denoted by $\hat{Z} \subset V$. For a point $\alpha \in Z$, the affine tangent space of $Z$ at $\alpha$ is

$$
\hat{T}_{\alpha}(Z):=T_{u}(\hat{Z}) \subset V \text { for a non-zero vector } u \in \hat{\alpha} .
$$

This is independent of the choice of $u$.

Definition 3.2. - A cone structure on a complex manifold $M$ is a submanifold $\mathscr{C} \subset \mathbb{P} T(M)$ such that the projection $\varpi: \mathscr{C} \rightarrow M$ is a smooth morphism with connected fibers. For each point $x \in M$, the fiber $\varpi^{-1}(x)$ will be denoted by $\mathscr{C}_{x}$. Associated to a cone structure are three natural distributions $V \subset \mathcal{J} \subset \mathscr{P}$ on $\mathscr{C}$ whose fiber at $\alpha \in \mathscr{C}_{x}$ is defined as follows in terms of the differential $d \varpi_{\alpha}: T_{\alpha}(\mathscr{C}) \rightarrow T_{x}(M)$ of $\varpi$ at $\alpha$ :

$$
V_{\alpha}:=d \varpi_{\alpha}^{-1}(0), \mathcal{T}_{\alpha}:=d \varpi_{\alpha}^{-1}(\hat{\alpha}), \mathscr{P}_{\alpha}:=d \varpi_{\alpha}^{-1}\left(\hat{T}_{\alpha}\left(\mathscr{C}_{x}\right)\right) .
$$

Of course, $V \subset T(\mathscr{C})$ is the relative tangent bundle of $\varpi$.

Definition 3.3. - Given a cone structure $\mathscr{C} \subset \mathbb{P} T(M)$ and the associated distributions $V \subset \mathcal{T} \subset \mathscr{P}$, a line subbundle $\mathcal{F} \subset T(\mathscr{C})$, with locally free quotient $T(\mathscr{C}) / \mathcal{F}$, is called a connection (or a conic connection) if $\mathcal{F} \subset \mathcal{T}$ and $\mathcal{F} \cap \mathcal{V}=0$, i.e., it splits the exact sequence

$$
0 \longrightarrow V \longrightarrow \mathcal{T} \longrightarrow \mathcal{T} / \mathcal{V} \cong L^{*} \longrightarrow 0
$$


where $L$ denotes the restriction of the relative hyperplane bundle on $\mathbb{P} T(M)$. A connection $\mathcal{F} \subset \mathcal{J}$ is $\mathscr{P}_{\text {-splitting }}$ if the sequence

$$
0 \longrightarrow V \longrightarrow \mathscr{P} / \mathcal{F} \longrightarrow \mathscr{P} / \mathcal{T} \longrightarrow 0
$$

splits.

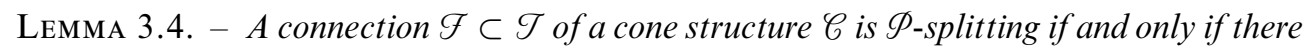
exists a vector subbundle $W \subset \mathscr{P}$ with an isomorphism $\mathscr{D} / \mathcal{V} \cong \mathcal{W}$ splitting

$$
0 \longrightarrow V \longrightarrow \mathscr{P} \longrightarrow \mathscr{P} / \mathcal{V} \longrightarrow 0
$$

such that $\mathcal{F}=W \cap \mathcal{T}$.

Proof. - If such a $\mathcal{W}$ exists, $W / \mathcal{F} \subset \mathscr{P} / \mathcal{F}$ splits the sequence (3.1). Conversely, the splitting of the sequence (3.1) induces a splitting of (3.2) by $W \subset \mathscr{P}$ satisfying $\mathcal{F}=W \cap \mathcal{T}$.

Proposition 3.5. - Given a cone structure $\mathscr{C} \subset \mathbb{P} T(M)$ and a point $x \in M$, set $V=T_{x}(M)$. Denote by $Z \subset \mathbb{P} V$ the projective submanifold $\mathscr{C}_{x} \subset \mathbb{P} T_{x}(M)$.

(a) When there exists a conic connection $\mathcal{F}$, the extension class of

$$
0 \longrightarrow V \longrightarrow T(\mathscr{C}) / \mathcal{F} \longrightarrow T(\mathscr{C}) / \mathcal{T} \longrightarrow 0
$$

restricted to $Z$ lies in $H^{1}\left(Z,\left.T(Z) \otimes T^{*}(\mathbb{P} V)\right|_{Z} \otimes L\right)$.

(b) When a conic connection $\mathcal{F}$ is $\mathscr{P}$-splitting, given by $\mathscr{P}=\mathcal{V} \oplus$ in Lemma 3.4, the extension class of

$$
0 \longrightarrow V \longrightarrow T(\mathscr{C}) / \mathcal{W} \longrightarrow T(\mathscr{C}) / \mathscr{P} \longrightarrow 0
$$

restricted to $Z$ lies in $H^{1}\left(Z, T(Z) \otimes N_{Z}^{*} \otimes L\right)$.

Proof. - At a point $\alpha \in Z$,

$$
T_{\alpha}(\mathscr{C}) / \mathcal{T}_{\alpha}=T_{\alpha}(\mathscr{C}) / d \varpi_{\alpha}^{-1}(\hat{\alpha})=V / \hat{\alpha}=\hat{\alpha} \otimes T_{\alpha}(\mathbb{P} V) .
$$

Thus the restriction of $T(\mathscr{C}) / \mathcal{J}$ to $Z$ is naturally isomorphic to $\left.T(\mathbb{P} V)\right|_{Z} \otimes L^{*}$, showing (a). Similarly,

$$
T_{\alpha}(\mathscr{C}) / \mathscr{P}_{\alpha}=T_{\alpha}(\mathscr{C}) / d \varpi_{\alpha}^{-1}\left(\hat{T}_{\alpha}(Z)\right)=V / \hat{T}_{\alpha}(Z)=\hat{\alpha} \otimes N_{Z, \alpha} .
$$

Thus the restriction of $T(\mathscr{C}) / \mathscr{P}$ to $Z$ is naturally isomorphic to $N_{Z} \otimes L^{*}$, showing (b).

Proposition 3.6. - Given a cone structure $\mathscr{C} \subset \mathbb{P} T(M)$ and a point $x \in M$, set $V=T_{x}(M)$ and denote by $Z \subset \mathbb{P} V$ the projective submanifold $\mathscr{C}_{x} \subset \mathbb{P} T_{x}(M)$. Let $\kappa \in H^{1}(Z, T(Z)) \otimes V^{*}$ be the the extension class of the sequence

$$
0 \longrightarrow V \longrightarrow T(\mathscr{C}) \longrightarrow T(\mathscr{C}) / V=\varpi^{*} T(M) \longrightarrow 0
$$

restricted to $Z$. This is the Kodaira-Spencer class of $\varpi: \mathscr{C} \rightarrow M$ at $x$. Denote by $\widetilde{V}$ the trivial vector bundle on $Z$ with a fiber $V$. Consider two homomorphisms

$$
H^{1}\left(Z, T(Z) \otimes N_{Z}^{*} \otimes L\right) \stackrel{\sigma}{\rightarrow} H^{1}\left(Z,\left.T(Z) \otimes T^{*}(\mathbb{P} V)\right|_{Z} \otimes L\right) \stackrel{\epsilon}{\rightarrow} H^{1}(Z, T(Z)) \otimes V^{*}
$$

where $\sigma$ is the last homomorphism of the exact sequence in Proposition 2.2 and $\epsilon$ is induced by the Euler sequence

$$
\left.0 \rightarrow T^{*}(\mathbb{P} V)\right|_{Z} \otimes L \rightarrow \widetilde{V}^{*} \rightarrow L \rightarrow 0
$$




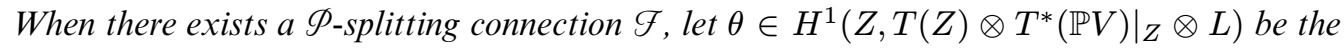
extension class in Proposition 3.5 (a) and let $\xi \in H^{1}\left(Z, T(Z) \otimes N_{Z}^{*} \otimes L\right)$ be the extension class in Proposition 3.5 (b). Then

$$
\kappa=\epsilon(\theta) \text { and } \theta=\sigma(\xi) .
$$

Proof. - If there exists a conic connection $\mathcal{F}$, i.e., $\mathcal{T}=\mathcal{V} \oplus \mathcal{F}$, the extension class of (3.3) comes from the extension class of Proposition 3.5 (a). Thus $\kappa=\epsilon(\theta)$. If $\mathcal{F}$ is $\mathscr{D}$-splitting, then the extension class of Proposition 3.5 (a) comes from the extension class of Proposition 3.5 (b). Thus $\theta=\sigma(\xi)$.

Proposition 3.7. - Let $\mathscr{C} \subset \mathbb{P} T(M)$ be a cone structure admitting a $\mathscr{D}_{\text {-splitting conic }}$ connection $\mathcal{F} \subset \mathcal{T}$. If $H^{0}\left(\mathscr{C}_{x}, T\left(\mathscr{C}_{x}\right)\right)=0$ and

$$
\operatorname{dim} H^{1}\left(\mathscr{C}_{x}, T\left(\mathscr{C}_{x}\right) \otimes N_{\mathscr{C}_{x}}^{*} \otimes L\right) \leq \operatorname{dim} H^{0}\left(\mathscr{C}_{x}, L\right),
$$

then the Kodaira-Spencer map $\kappa_{x}: T_{x}(M) \rightarrow H^{1}\left(\mathscr{C}_{x}, T\left(\mathscr{C}_{x}\right)\right)$ associated to the family $\varpi: \mathscr{C} \rightarrow M$ vanishes, i.e., the sequence (3.3) in Proposition 3.6 splits in a neighborhood of $\mathscr{C}_{x}$. Furthermore, this splitting $\varpi^{*} T(M) \subset T(\mathscr{C})$ can be chosen such that its intersection with the subbundle $\mathcal{T} \subset T(\mathscr{C})$ coincides with the splitting of

$$
0 \longrightarrow V \longrightarrow \mathcal{T} \longrightarrow \mathcal{T} / \mathcal{V} \cong L^{*} \longrightarrow 0
$$

in Definition 3.3 given by the conic connection $\mathcal{F}$.

Proof. - Applying Proposition 2.2 to $Z=\mathscr{C}_{x}$, the homomorphism $\sigma$ vanishes. Thus Proposition 3.6 implies the result.

Now we recall some definitions and results from [5].

Definition 3.8. - Let $M$ be a complex manifold of dimension $n$. Fix a vector space $V$ of dimension $n$. A $V$-valued 1-form $\omega$ on $M$ is called a coframe if for each $x \in M$, the homomorphism $\omega_{x}: T_{x}(M) \rightarrow V$ is an isomorphism.

Definition 3.9. - Let $V$ be an $n$-dimensional vector space and let $Z \subset \mathbb{P} V$ be a fixed non-singular projective subvariety. A cone structure $\mathscr{C} \subset \mathbb{P} T(M)$ on an $n$-dimensional manifold $M$ is said to be $Z$-isotrivial if for each $x \in M$, the inclusion $\left(\mathscr{C}_{x} \subset \mathbb{P} T_{x}(M)\right)$ is isomorphic to $(Z \subset \mathbb{P} V)$ up to projective transformations.

Definition 3.10. - Given $Z \subset \mathbb{P} V$ and a $Z$-isotrivial cone structure on $M$, a coframe $\omega$ on $M$ is said to be adapted to the cone structure if for each $x \in M$, the isomorphism $\omega_{x}: T_{x}(M) \rightarrow V$ sends $\mathscr{C}_{x} \subset \mathbb{P} T_{x}(M)$ to $Z \subset \mathbb{P} V$. Given any $Z$-isotrivial cone structure on a manifold $M$, an adapted coframe exists if we shrink $M$.

Proposition 3.11. - Given a coframe $\omega$ adapted to a Z-isotrivial cone structure $\mathscr{C} \subset \mathbb{P} T(M)$, let $\tau: \mathbb{P} T(M) \rightarrow \mathbb{P} V$ and $\gamma:=\left.\tau\right|_{\mathscr{C}}: \mathscr{C} \rightarrow Z$ be the submersive holomorphic maps induced by $\omega$. Then $\Gamma:=\operatorname{Ker}(d \gamma) \cap \mathcal{J}$ is a connection on $\mathscr{C}$, called the geodesic connection of the adapted coframe $\omega$.

$4^{\text {e }}$ SÉRIE - TOME $46-2013-\mathrm{N}^{\mathrm{o}} 4$ 
Proof. - This is proved in [5] using a slightly different terminology. A coframe induces a natural vector field on $T(M)$ called its geodesic flow (Definition 2.9 of [5]). If the coframe is adapted to a cone structure $\mathscr{C} \subset \mathbb{P} T(M)$ the geodesic flow is tangent to the homogeneous cone $\hat{\mathscr{C}} \subset T(M)$, inducing a holomorphic foliation $\Gamma \subset T(\mathscr{C})$ of rank 1 on $\mathscr{C}$. This is exactly $\operatorname{Ker}(d \gamma) \cap \mathcal{J}$. That $\Gamma$ is a connection is Proposition 5.4 of [5].

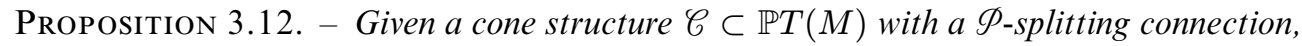
suppose that for each $x \in M, H^{0}\left(\mathscr{C}_{x}, T\left(\mathscr{C}_{x}\right)\right)=0$ and

$$
\operatorname{dim} H^{1}\left(\mathscr{C}_{x}, T\left(\mathscr{C}_{x}\right) \otimes N_{\mathscr{C}_{x}}^{*} \otimes L\right) \leq \operatorname{dim} H^{0}\left(\mathscr{C}_{x}, L\right)
$$

so that the Kodaira-Spencer map vanishes at every point by Proposition 3.7. Assume furthermore that the cone structure is $Z$-isotrivial for some $Z \subset \mathbb{P} V$. Then the geodesic connection of an

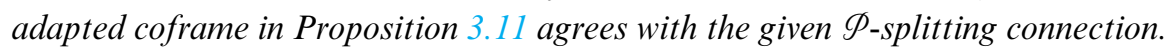

Proof. - Since $H^{0}\left(\mathscr{C}_{x}, T\left(\mathscr{C}_{x}\right)\right)=0$, there is a unique splitting of (3.3). From Proposition 3.7, the splitting of (3.3) respects the given $\mathscr{P}$-splitting conic connection $\mathcal{F}$. The tangent spaces to the fibers of the map $\gamma$ in Proposition 3.11 induced by any adapted coframe give a splitting of (3.3). Thus the geodesic connection $\Gamma$ of Proposition 3.11 must agree with the given $\mathscr{P}$-splitting connection $\mathcal{F}$.

Definition 3.13. - A conic connection $\mathcal{F} \subset T(\mathscr{C})$ is a characteristic connection if for any local section $v$ of $\mathscr{P}$ and any local section $w$ of $\mathcal{F}$, both regarded as local vector fields on the manifold $\mathscr{C}$, the Lie bracket $[v, w]$ is a local section of $\mathscr{P}$ again.

Definition 3.14. - Given a non-singular projective variety $Z \subset \mathbb{P} V$, the flat $Z$-isotrivial cone structure is the cone structure $Z \subset \mathbb{P} T(V)$ on the manifold $V$, given by $V \times Z$ under the natural trivialization $\mathbb{P} T(V) \cong V \times \mathbb{P} V$. A cone structure $\mathscr{C} \subset \mathbb{P} T(M)$ is locally flat at $x$ if there exist a neighborhood $U$ of $x$ and an unramified injective holomorphic map $\varphi: U \rightarrow V$ such that the differential $d \varphi: \mathbb{P} T(U) \rightarrow \mathbb{P} T(V)$ sends the cone structure $\mathscr{C} \cap \mathbb{P} T(U)$ onto $Z \cap \mathbb{P} T(\varphi(U))$.

The following is Theorem 5.8 in [5].

Theorem 3.15. - Let $Z \subset \mathbb{P V}$ be a non-singular variety such that

(a) $Z$ is linearly normal;

(b) $Z$ is tangentially non-degenerate; and

(c) $H^{0}\left(Z,\left.\operatorname{End}^{0}(T(Z)) \otimes \Theta(1)\right|_{Z}\right)=0$.

Let $\mathscr{C} \subset \mathbb{P} T(M)$ be a $Z$-isotrivial cone structure with an adapted coframe $\omega$. If the geodesic connection $\Gamma$ in Proposition 3.11 induced by $\omega$ on $\mathscr{C}$ is a characteristic connection, then the cone structure is locally flat.

As a conclusion of the discussion in this section, we obtain

Theorem 3.16. - Let $\mathscr{C} \subset \mathbb{P} T(M)$ be a cone structure with a $\mathscr{P}_{\text {-splitting characteristic }}$ connection $\mathcal{F} \subset T(\mathscr{C})$. Assume that for each $x \in M$, the submanifold $\mathscr{C}_{x} \subset \mathbb{P} T_{x}(M)$ satisfies

(1) $\mathscr{C}_{x}$ is linearly normal;

(2) $\mathscr{C}_{x}$ is tangentially non-degenerate;

(3) $H^{0}\left(\mathscr{C}_{x}, T\left(\mathscr{C}_{x}\right)\right)=0$; 
(4) $H^{1}\left(\mathscr{C}_{x}, \vartheta_{\mathscr{C}_{x}}\right)=0$; and

(5) $\operatorname{dim} H^{1}\left(\mathscr{C}_{x}, T\left(\mathscr{C}_{x}\right) \otimes N_{\mathscr{C}_{x}}^{*} \otimes L\right) \leq \operatorname{dim} H^{0}\left(\mathscr{C}_{x}, L\right)$.

Then the cone structure is locally flat.

Proof. - By (3) and (5), Proposition 3.7 implies that the fibers of the family $\varpi: \mathscr{C} \rightarrow M$ of complex manifolds are all biholomorphic to each other. By (1) and (4), the family of projective varieties $\left\{\mathscr{C}_{x} \subset \mathbb{P} T_{x}(M), x \in M\right\}$ are all isomorphic up to projective equivalence. It follows that the cone structure is $Z$-isotrivial for some fixed projective variety $Z \subset \mathbb{P} V$. This $Z \subset \mathbb{P} V$ satisfies the conditions (a) and (b) of Theorem 3.15 from (1) and (2). From (3) and (5), Proposition 2.2 implies that $Z$ satisfies also the condition (c) of Theorem 3.15. It follows that $\mathscr{C}$ is locally flat.

\section{4. $\mathscr{P}$-splitting property of the characteristic connection on the VMRT-structure}

Let $X$ be a uniruled projective manifold and $\mathcal{K}$ be a family of minimal rational curves as defined in Section 1. Let $\rho: \mathcal{U} \rightarrow \mathcal{K}$ be the universal $\mathbb{P}^{1}$-bundle and $\mu: \mathcal{U} \rightarrow X$ be the evaluation morphism such that the $\mu$-images of the $\mathbb{P}^{1}$-fibers of $\rho$ are minimal rational curves of $X$ belonging to $\mathcal{K}$. For a general point $x \in X$, the normalization of the space $\mathcal{K}_{x} \subset \mathcal{K}$ of members passing through $x$ is a non-singular projective variety (cf. [11] Section II.3 or [9]). To simplify the notation, we will use $\mathcal{K}_{x}$ from now on to denote its normalization. We denote by $\tau_{x}: \mathcal{K}_{x} \rightarrow \mathscr{C}_{x}$ the tangent morphism at $x$, which sends a member of $\mathcal{K}_{x}$ to its tangent direction at $x$. This is a well-defined morphism by Theorem 3.4 of [9]. The following is a direct consequence of Proposition 1.4 of [4] and Theorem 1 of [8].

Proposition 4.1. - In the above setting, suppose that the assumption (*) in Section 1 holds, i.e., the VMRT $\mathscr{C}_{x}$ is smooth for a general point $x \in X$. Then

1. the tangent morphism $\tau_{x}: \mathcal{K}_{x} \rightarrow \mathscr{C}_{x}$ is biregular, and

2. each member of $\mathcal{K}_{x}$ is a standard rational curve, i.e., for each member $[C] \in \mathcal{K}_{x}$, under the morphism $\nu:=\left.\mu\right|_{\rho^{-1}([C])}: \mathbb{P}^{1} \rightarrow C \subset X$,

$$
\nu^{*} T(X) \cong \theta(2) \oplus \theta(1)^{p} \oplus \theta^{n-1-p}
$$

where $n=\operatorname{dim} X$ and $p=\operatorname{dim} \mathscr{C}_{x}=\operatorname{dim} \mathcal{K}_{x}$.

Let us make the assumption $\left(^{*}\right)$ and assume furthermore that $\mathscr{C}_{x}$ is irreducible, for simplicity. Then there exists a Zariski open subset $X^{o} \subset X$ such that $\mathscr{C} \cap \mathbb{P} T\left(X^{o}\right)$ is a cone structure on $X^{o}$.

ExAmple 4.2. - In Example 1.1, set

$$
X^{o}:=\beta^{-1}\left(\mathbb{P}^{n} \backslash \mathbb{P}^{n-1}\right) \subset X_{Z} .
$$

The VMRT-structure $\mathscr{C} \subset \mathbb{P} T\left(X_{Z}\right)$ of $\mathcal{K}_{Z}$ defines a cone structure on $X^{o}$. In fact, under the natural biregular morphism $X^{o} \cong \mathbb{C}^{n}$, the cone structure is the flat $Z$-isotrivial cone structure in Definition 3.14.

The cone structure $\mathscr{C}$ on $X^{o}$ is equipped with a natural characteristic connection: 
Proposition 4.3. - Let $X$ be a uniruled projective manifold with a family of minimal rational curves such that $\mathscr{C}_{x}$ at a general point $x \in X$ is smooth and irreducible. Choose a Zariski open subset $X^{\circ} \subset X$ as explained above. After shrinking $X^{o}$ if necessary, we have a biregular morphism

$$
\tau: \mu^{-1}\left(X^{o}\right) \rightarrow \mathscr{C} \cap \mathbb{P} T\left(X^{o}\right)
$$

which is defined by collecting the biregular morphisms $\tau_{x}$ in Proposition 4.1. Then the fibers of the universal family morphism $\rho$ are sent by $\tau$ to a connection $\mathcal{F}$ on the cone structure $\mathscr{C} \cap \mathbb{P} T\left(X^{o}\right)$. This $\mathcal{F}$ is a characteristic connection of the cone structure.

Proof. - By the definition of $\tau$, if $F \subset \mu^{-1}\left(X^{o}\right)$ is a germ of a fiber of $\rho$, then $\tau(F)$ is exactly $\mathbb{P} T(\mu(F))$. This means that the fibers of $\rho$ are sent by $\tau$ to a connection $\mathcal{F}$ of the cone structure $\mathscr{C}$. That $\mathcal{F}$ is a characteristic connection is proved in Proposition 8 of [8].

For the proof of the next proposition, we recall the following notion.

Definition 4.4. - For an effective 0 -cycle $H=p_{1}+\cdots+p_{m}$ on the affine line $\mathbb{A}^{1}$, its center of mass is the point $\bar{H} \in \mathbb{A}^{1}$ defined by the average $\left(p_{1}+\cdots+p_{m}\right) / m$ after an identification $\mathbb{A}^{1} \cong \mathbb{C}$ by fixing a base point $0 \in \mathbb{A}^{1}$. The definition of $\bar{H}$ is independent of the choice of the base point.

Proposition 4.5. - In the setting of Proposition 4.3, there exists a neighborhood $M \subset X$ of a general point $x \in X$ such that for the cone structure $\mathscr{C} \cap \mathbb{P} T(M)$, the characteristic connection in Proposition 4.3 is $\mathscr{P}_{\text {-splitting. }}$

Proof. - Let $\mathcal{F} \subset \mathcal{J}$ be the connection from Proposition 4.3. For a general point $x \in X^{o}$, we have an exact sequence of vector bundles on $\mathscr{C}_{x}$

$$
\left.\left.0 \longrightarrow T\left(\mathscr{C}_{x}\right) \longrightarrow(\mathscr{P} / \mathcal{F})\right|_{\mathscr{C}_{x}} \longrightarrow(\mathscr{P} / \mathcal{T})\right|_{\mathscr{C}_{x}} \longrightarrow 0
$$

coming from (3.1). To prove the proposition, it suffices to show the splitting of (4.1).

Let $\rho_{x}: \mathcal{U}_{x} \rightarrow \mathcal{K}_{x}$ be the universal $\mathbb{P}^{1}$-bundle with the evaluation morphism $\mu_{x}: \mathcal{U}_{x} \rightarrow X$ with a distinguished section $E_{x}=\mu_{x}^{-1}(x)$ of $\rho_{x}$. We claim that there is a section $E_{x}^{\prime} \subset \mathcal{U}_{x}$ of $\rho_{x}$ with $E_{x}^{\prime} \cap E_{x}=\varnothing$.

To prove the claim, fix an ample hypersurface $H \subset X$ with $x \notin H$. Let $H_{x} \subset \mathcal{U}_{x}$ be the inverse image of $H$ under $\mu_{x}$. Then $H_{x}$ is a hypersurface in $\mathcal{U}_{x}$ flat over $\mathcal{K}_{x}$ and disjoint from $E_{x}$. The bundle $\rho_{x}^{o}: \mathcal{U}_{x} \backslash E_{x} \rightarrow \mathcal{K}_{x}$ is an affine bundle of rank 1. For each point $\alpha \in E_{x}$, let $\alpha^{\prime} \in \mathcal{U}_{x} \backslash E_{x}$ be the center of mass, in the sense of Definition 4.4, of the 0-cycle defined by the scheme-theoretic intersection $\left(\rho_{x}^{o}\right)^{-1}\left(\rho_{x}(\alpha)\right) \cap H_{x}$. Then the collection $\left\{\alpha^{\prime}, \alpha \in E_{x}\right\}$ defines a hypersurface $E_{x}^{\prime} \subset \mathcal{U}_{x} \backslash E_{x}$ which is biregular to $\mathcal{K}_{x} \cong E_{x}$ by the restriction of the morphism $\rho_{x}$.

Now identify $E_{x}$ with $\mathscr{C}_{x}$ via the biregular morphism $\tau_{x}$ in Proposition 4.1. For a point $\alpha \in \mathscr{C}_{x}=E_{x}$, define

$$
\mu_{\alpha}:=\left.\mu_{x}\right|_{\rho_{x}^{-1}\left(\rho_{x}(\alpha)\right)}: \rho_{x}^{-1}\left(\rho_{x}(\alpha)\right) \rightarrow X
$$

Then $\mu_{\alpha}$ is an immersion of $\mathbb{P}^{1}$ such that

$$
\mu_{\alpha}^{*} T(X) \cong \theta(2) \oplus \theta(1)^{p} \oplus \theta^{n-1-p}
$$

where $p=\operatorname{dim} \mathscr{C}_{x}$ and $n=\operatorname{dim} X$ from Proposition 4.1. Let $T^{\alpha} \subset \mu_{\alpha}^{*} T(X)$ be the $\Theta(2)$-factor corresponding to the image of $T\left(\rho_{x}^{-1}\left(\rho_{x}(\alpha)\right)\right)$. Let $T^{+} \subset \mu_{\alpha}^{*} T(X)$ be the 
subbundle corresponding to the factor $\Theta(2) \oplus \Theta(1)^{p}$. Denote by $\mathbf{m}_{\alpha}$ the maximal ideal of the point $\alpha$ on $\rho_{x}^{-1}\left(\rho_{x}(\alpha)\right)$. In terms of sections of the bundles $T^{\alpha} \subset T^{+}$on the curve $\rho_{x}^{-1}\left(\rho_{x}(\alpha)\right)$, there are canonical identifications (cf. Proof of Proposition 8 in [8])

$$
\begin{aligned}
T_{\alpha}(\mathscr{C}) & =H^{0}\left(\mu_{\alpha}^{*} T(X)\right) / H^{0}\left(T^{\alpha} \otimes \mathbf{m}_{\alpha}\right) \\
V_{\alpha} & =H^{0}\left(T^{+} \otimes \mathbf{m}_{\alpha}\right) / H^{0}\left(T^{\alpha} \otimes \mathbf{m}_{\alpha}\right) \\
\mathcal{F}_{\alpha} & =H^{0}\left(T^{\alpha}\right) / H^{0}\left(T^{\alpha} \otimes \mathbf{m}_{\alpha}\right) \\
\mathscr{P}_{\alpha} & =H^{0}\left(T^{+}\right) / H^{0}\left(T^{\alpha} \otimes \mathbf{m}_{\alpha}\right) .
\end{aligned}
$$

It follows that

$$
\begin{aligned}
& (\mathscr{P} / \mathcal{F})_{\alpha}=H^{0}\left(T^{+}\right) / H^{0}\left(T^{\alpha}\right) \\
& (\mathscr{P} / \mathcal{T})_{\alpha}=H^{0}\left(T^{+}\right) /<H^{0}\left(T^{\alpha}\right), H^{0}\left(T^{+} \otimes \mathbf{m}_{\alpha}\right)>.
\end{aligned}
$$

For $\alpha^{\prime} \in E_{x}^{\prime}$ defined in the claim above, denote by $\mathbf{m}_{\alpha^{\prime}}$ the maximal ideal at $\alpha^{\prime}$ and define $\phi_{\alpha} \subset(\mathscr{P} / \mathcal{F})_{\alpha}$ by

$$
\phi_{\alpha}:=H^{0}\left(T^{+} \otimes \mathbf{m}_{\alpha^{\prime}}\right) /\left(H^{0}\left(T^{+} \otimes \mathbf{m}_{\alpha^{\prime}}\right) \cap H^{0}\left(T^{\alpha}\right)\right) .
$$

Note

$$
H^{0}\left(T^{+} \otimes \mathbf{m}_{\alpha} \otimes \mathbf{m}_{\alpha^{\prime}}\right)=H^{0}\left(T^{\alpha} \otimes \mathbf{m}_{\alpha} \otimes \mathbf{m}_{\alpha^{\prime}}\right)
$$

Thus

$$
\S_{\alpha} \cap V_{\alpha}=H^{0}\left(T^{+} \otimes \mathbf{m}_{\alpha} \otimes \mathbf{m}_{\alpha^{\prime}}\right) /\left(H^{0}\left(T^{\alpha} \otimes \mathbf{m}_{\alpha}\right) \cap H^{0}\left(T^{+} \otimes \mathbf{m}_{\alpha} \otimes \mathbf{m}_{\alpha^{\prime}}\right)\right)=0 .
$$

Furthermore $H^{0}\left(T^{+}\right) / H^{0}\left(T^{\alpha}\right)$ is spanned by the images of $H^{0}\left(T^{+} \otimes \mathbf{m}_{\alpha}\right)$ and $H^{0}\left(T^{+} \otimes \mathbf{m}_{\alpha^{\prime}}\right)$, because

$$
H^{0}\left(\mathbb{P}^{1}, \oslash(1)\right)=H^{0}\left(\mathbb{P}^{1}, \oslash(1) \otimes \mathbf{m}_{0}\right)+H^{0}\left(\mathbb{P}^{1}, \oslash(1) \otimes \mathbf{m}_{\infty}\right)
$$

for any two distinct points $0, \infty \in \mathbb{P}^{1}$. This means that

$$
(\mathscr{P} / \mathcal{F})_{\alpha}=V_{\alpha} \oplus \phi_{\alpha}
$$

It follows that

$$
\left\{\varnothing_{\alpha} \subset(\mathscr{P} / \mathcal{F})_{\alpha}, \alpha \in E_{x}\right\}
$$

defines a vector subbundle of $\left.(\mathscr{P} / \mathcal{F})\right|_{\mathscr{C}_{x}}$ splitting the sequence (4.1).

Proof of Theorem 1.9 and Theorem 1.10. - Theorem 1.10 is a direct consequence of Theorem 3.16 and Proposition 4.5. Theorem 1.9 is a corollary of Theorem 1.10 via Proposition 2.4 .

\section{Proof of Theorem 1.4}

In this section, we will derive Theorem 1.4 from Theorem 1.9. The overall strategy is the following. By Theorem 1.9, we know that the VMRT-structure of $X$ is $Z$-isotrivial for some hypersurface $Z \subset \mathbb{P}^{n-1}$ and locally flat. We will use this to construct a dominant rational map $\Psi$ from $X_{Z}$ to $X$, which sends general members of $\mathcal{K}_{Z}$ to those of $\mathcal{K}$. This construction is done by modifying the arguments in [7]. After we obtain $\Psi$, we prove that $\Psi$ is equivariant with respect to an action of a finite group $G$ on $X_{Z}$ and factors through a birational map $\Phi: X_{Z} / G \rightarrow X$.

$4^{\mathrm{e}}$ SÉRIE - TOME $46-2013-\mathrm{N}^{\circ} 4$ 
Let us begin with the construction of $\Psi: X_{Z} \rightarrow X$. We will follow the arguments in [7] step-by-step and indicate what modifications should be made.

Proposition 5.1. - Let $X^{1}$ (resp. $X^{2}$ ) be a uniruled projective manifold with a family $\mathcal{K}^{1}$ (resp. $\mathcal{K}^{2}$ ) of minimal rational curves. Assume that the VMRT $\mathscr{C}_{x^{1}}^{1} \subset \mathbb{P} T_{x^{1}}\left(X^{1}\right)$ (resp. $\mathscr{C}_{x^{2}}^{2} \subset \mathbb{P} T_{x^{2}}\left(X^{2}\right)$ ) at a general point $x^{1} \in X^{1}$ (resp. $x^{2} \in X^{2}$ ) is smooth, irreducible and non-degenerate. Suppose there exist an analytic neighborhood $x^{1} \in U^{1} \subset X^{1}$ (resp. $\left.x^{2} \in U^{2} \subset X^{2}\right)$ and a biholomorphic map $\psi: U^{1} \rightarrow U^{2}$, which makes the VMRT-structure at $x^{1}$ and the VMRT-structure at $x^{2}$ equivalent in the sense of Definition 1.6. Then

(1) for a general curve $C^{1} \subset X^{1}$ belonging to $\mathcal{K}_{x^{1}}^{1}$, there exist a member $C^{2} \subset X^{2}$ of $\mathcal{K}_{x^{2}}^{2}$, an analytic neighborhood $C^{1} \subset W^{1}$ (resp. $C^{2} \subset W^{2}$ ) and a meromorphic map $\varphi: W^{1} \rightarrow W^{2}$ such that $\left.\varphi\right|_{U_{1} \cap W_{1}}=\left.\psi\right|_{U_{1} \cap W_{1}}$; and

(2) there exists a generically finite morphism $p: \tilde{X}^{1} \rightarrow X^{1}$ from a projective variety $\tilde{X}^{1}$ which is etale over a Zariski open subset $X_{o}^{1} \subset X^{1}$ and a dominant meromorphic map $\Psi: p^{-1}\left(X_{o}^{1}\right) \rightarrow X^{2}$ extending $\varphi$.

Proof. - (1) is contained in Proposition 2.1 of [7]. It is stated there under the additional assumption that $\operatorname{Pic}\left(X^{1}\right) \cong \operatorname{Pic}\left(X^{2}\right) \cong \mathbb{Z}$, but its proof does not use that assumption. (2) is contained in Proposition 3.4 of [7], which is also stated with the assumption $\operatorname{Pic}\left(X^{1}\right) \cong \operatorname{Pic}\left(X^{2}\right) \cong \mathbb{Z}$. This assumption is needed only for the proof of Lemma 3.1 in [7], which claims that if $x$ is a general point of $X^{1}$ then for any irreducible subvariety $A \subset X$ containing $x$ and any general point $y \in A$, there exists a member $C$ of $\mathcal{K}^{1}$ with $y \in C$ and $C \not \subset A$. But this claim is obvious in our case, from our assumption that the VMRT $\mathscr{C}_{y}^{1}$ at a general point $y \in A$ is non-degenerate.

For the next step, we need the following property of $X_{Z}$.

Lemma 5.2. - Fix a hyperplane $H \subset \mathbb{P}^{n}, n \geq 3$ and a smooth hypersurface $Z \subset H$ of degree $m \geq 2$. Let $D \subset \mathbb{P}^{n}$ be an irreducible hypersurface different from $H$. Let $X_{Z} \rightarrow \mathbb{P}^{n}$ be the blow-up of $\mathbb{P}^{n}$ along $Z$ and let $\bar{D} \subset X_{Z}$ be the proper image of $D$. Then for a general line $\ell \subset \mathbb{P}^{n}$ intersecting $Z$, its proper image $\bar{\ell} \subset X_{Z}$ intersects $\bar{D}$.

Proof. - This is obvious if $Z \not \subset D$. Let us assume that $Z \subset D$.

Fix a general plane $P \cong \mathbb{P}^{2} \subset \mathbb{P}^{n}$ such that $Z^{\prime}:=Z \cap P$ consists of $m$ distinct points, say $\left\{z_{1}, \ldots, z_{m}\right\} . Z^{\prime}$ is contained in the intersection of the line $H^{\prime}:=H \cap P$ and the irreducible plane curve $D^{\prime}:=D \cap P$. The degree $d$ of $D^{\prime}$ must be strictly bigger than the multiplicity mult $_{z_{1}}\left(D^{\prime}\right)$ of $D^{\prime}$ at $z_{1}$. If we choose a general line $\ell \subset P$ through the point $z_{1}$, the local intersection number of $\ell$ with $D^{\prime}$ at $z_{1}$ is precisely mult $z_{z_{1}}\left(D^{\prime}\right)<d$. Thus $\ell$ intersects $D^{\prime}$ at some point other than $z_{1}$. Since $\ell$ is disjoint from $\left\{z_{2}, \ldots, z_{m}\right\}$, we see that $\ell$ intersects $D$ at a point outside $Z$. This implies that $\bar{\ell}$ intersects $\bar{D}$.

The following lemma is to replace Lemma 4.2 in [7] in our setting.

Lemma 5.3. - Let $Z \subset \mathbb{P}^{n-1}$ be a smooth hypersurface of degree $\geq 2$ and let $X_{Z}$ be as in Example 1.1. Let $p: Y \rightarrow X_{Z}$ be a generically finite morphism from a normal projective variety $Y$ to $X_{Z}$. Assume that for a general member $C \subset X_{Z}$ of $\mathcal{K}_{Z}$, if a component $C^{\prime}$ of $p^{-1}(C)$ is dominant over $C$, then $C^{\prime}$ is birational to $C$ by $p$. Then $p$ is a birational map from $Y$ to $X_{Z}$. 
Proof. - By Lemma 2.1 of [4], $p^{-1}(C)$ is contained in the smooth locus of $Y$ for a general member $C$ of $\mathcal{K}_{Z}$. The assumption implies that for a component $C^{\prime}$ of $p^{-1}(C)$ dominant over $C$, local deformations of $C^{\prime}$ correspond to local deformations of $C$. Hence the canonical degrees of $C$ and $C^{\prime}$ are equal, i.e., $K_{Y} \cdot C^{\prime}=K_{X_{Z}} \cdot C$ (cf. Proof of Lemma 4.2 in [7]). Suppose that $p$ is not birational, i.e., has degree $>1$. We will derive a contradiction.

Since $X_{Z}$ is simply-connected, there exists a ramification divisor $R \subset Y$ such that $p(R) \subset X_{Z}$ is a non-zero divisor. We claim that $C \cap p(R)=\varnothing$. If not, some component $C^{\prime}$ of $p^{-1}(C)$ dominant over $C$ intersects $R$. This contradicts $K_{Y} \cdot C^{\prime}=K_{X_{Z}} \cdot C$.

By the claim, the divisor $p(R)$ in $X_{Z}$ is disjoint from a general member $C$ of $\mathcal{K}_{Z}$. Thus under the projection $\beta: X_{Z} \rightarrow \mathbb{P}^{n}$, the image $\beta(p(R))$ must be contained in the hyperplane $\mathbb{P}^{n-1} \subset \mathbb{P}^{n}$ by Lemma 5.2. This holds for any ramification divisor $R$ with $\operatorname{dim} \beta(p(R))=n-1$. It follows that $\beta \circ p: Y \rightarrow X_{Z} \rightarrow \mathbb{P}^{n}$ is unramified of degree $>1$ over $\mathbb{C}^{n}=\mathbb{P}^{n} \backslash \mathbb{P}^{n-1}$, a contradiction.

The next proposition corresponds to Proposition 4.3 of [7]. In fact, the two claims (i) and (ii) in its proof are exactly the 'two problems' mentioned at the end of the first paragraph in Section 4 of [7]. But its proof requires an argument different from the one in [7], because a hypersurface in $X_{Z}$ can be disjoint from minimal rational curves.

Proposition 5.4. - In the setting of Theorem 1.4, there exists a dominant rational map $\Psi: X_{Z} \rightarrow X$ whose differential sends the VMRT-structure of $X_{Z}$ to the VMRT-structure of $X$.

Proof. - Applying Proposition 5.1 (2) to $X^{1}=X_{Z}$ and $X^{2}=X$, we have a Zariski open subset $U \subset X_{Z}$, a generically finite morphism $p: \tilde{X}_{Z} \rightarrow X$ étale over $U$ and a dominant meromorphic map $\Psi: p^{-1}(U) \rightarrow X$ which induces an equivalence of VMRT-structures at a general point of $X_{Z}$ and a general point of $X$. It suffices to prove that (i) $\Psi$ is univalent, i.e., it descends to a meromorphic map $U \rightarrow X$, and (ii) $\Psi$ has no essential singularity along $X_{Z} \backslash U$, i.e., it extends to a rational map $X_{Z} \rightarrow X$.

(i) can be proved by the same argument as the proof of Proposition 4.1 in [7], by using our Lemma 5.3 in place of Lemma 4.2 of [7]. In particular, we can find a Zariski open subset $U \subset X_{Z}$ and regard $\Psi$ as a meromorphic map $\Psi: U \rightarrow X$.

To prove (ii), we recall Hartog's extension theorem that a meromorphic map defined outside an analytic subset of codimension 2 in a projective manifold can be extended to a rational map. Suppose there exists a hypersurface $D \subset X_{Z} \backslash U$. It suffices to show that $\Psi$ can be extended in a neighborhood of a general point of $D$.

If a general member of $\mathcal{K}_{Z}$ intersects $D$, then $\Psi$ can be extended in a neighborhood of a general point of $D$ by Proposition 5.1 (1). Thus we may assume that $D$ is disjoint from general members of $\mathcal{K}_{Z}$. By Lemma 5.2, this implies that $\beta(D)$ is the hyperplane $\mathbb{P}^{n-1} \subset \mathbb{P}^{n}$.

Fix a line $\ell \subset \mathbb{P}^{n}$ such that $\ell \not \subset \mathbb{P}^{n-1}$ and $\ell \cap Z=\varnothing$. Let $Y:=\mathrm{Bl}_{\ell}\left(\mathbb{P}^{n}\right) \rightarrow \mathbb{P}^{n}$ be the blow-up along $\ell$. Then we have a $\mathbb{P}^{2}$-bundle fibration

$$
\delta: Y \rightarrow B \cong \mathbb{P}^{n-2} .
$$

$4^{\text {e }}$ SÉRIE - TOME $46-2013$ - No 4 
Let $\bar{\ell} \subset X_{Z}$ be the proper transform of $\ell$ under $\beta: X_{Z} \rightarrow \mathbb{P}^{n}$. Let $Y^{\prime} \rightarrow X_{Z}$ be the blowup of $\bar{\ell}$. Then we have the induced morphism $Y^{\prime} \rightarrow Y$ giving rise to a surjective morphism $\gamma: Y^{\prime} \rightarrow B$ whose general fibers are $\mathbb{P}^{2}$ blown up at $m$ distinct points, $m=\operatorname{deg}(Z)$.

$$
\begin{aligned}
& B \stackrel{\gamma}{\leftarrow} Y^{\prime} \rightarrow X_{Z} \\
& \| \quad \quad \downarrow \quad \downarrow \beta \\
& B \stackrel{\delta}{\leftarrow} Y \rightarrow \mathbb{P}^{n} .
\end{aligned}
$$

Let $D^{\prime} \subset Y^{\prime}$ be the proper transform of $D$. It suffices to show that the meromorphic map $\Psi$, defined on the inverse image of $U$ in $Y^{\prime}$ can be extended in a neighborhood of a general point of $D^{\prime}$. Choose a small open subset $W \subset B$, such that $\gamma^{-1}(W)$ is the $\mathbb{P}^{2}$-bundle $\delta^{-1}(W)$ blown up at $m$ distinct sections. Then after blowing down $m-1$ of these sections, we get a complex manifold $Y$ with a fibration $\rho: Y \rightarrow W$ whose fiber $\rho^{-1}(w)$ over $w \in W$ is $\mathbb{P}^{2}$ blown up at one point, to be denoted by $\mu_{w}: \rho^{-1}(w) \rightarrow \mathbb{P}^{2}$. The image of $D^{\prime}$ in $y$ is a hypersurface $\mathscr{D} \subset \mathcal{Y}$ such that for each $w \in W, \rho^{-1}(w) \cap \mathscr{D}$ is the proper transform of a line in $\mathbb{P}^{2}$ passing through the blow-up center of $\mu_{w}: \rho^{-1}(w) \rightarrow \mathbb{P}^{2}$. Thus we can contract $\mathscr{D}$ in $\mathcal{Y}$ to get a $\mathbb{P}^{2}$-bundle $\bar{y} \rightarrow W$. Consider the restriction of the meromorphic map $\Psi$ on $\mathcal{Y} \backslash \mathscr{D}$. It descends to a meromorphic map defined on $\bar{y}$ outside a set of codimension 2, i.e., outside the contracted image of $\mathscr{D}$. Thus it can be extended to a meromorphic map on $\bar{y}$. This implies that $\Psi$ can be extended to a meromorphic map on $\mathcal{Y}$, and consequently on $\gamma^{-1}(W)$, which includes a neighborhood of a general point on $D^{\prime}$.

Now we need to get the finite group $G$ acting on $X_{Z}$. It is convenient to introduce the following notion.

Definition 5.5. - Given a cone structure $\mathscr{C} \subset \mathbb{P} T(M)$ on a complex manifold, a holomorphic vector field $v$ on a neighborhood of a point $x \in M$ preserves the cone structure if the natural lift of $v$ to a holomorphic vector field on $\mathbb{P} T(M)$, defined by integrating $v$ to local biholomorphisms, is tangent to the submanifold $\mathscr{C} \subset \mathbb{P} T(M)$.

The $\mathbb{C}^{*}$-action on $\mathbb{C}^{n}$ by scalar multiplication is generated by a vector field $E$ on the complex manifold $\mathbb{C}^{n}$, called the Euler vector field. In terms of a linear coordinate system $\left(z_{1}, \ldots, z_{n}\right)$ on $\mathbb{C}^{n}$

$$
E=z_{1} \frac{\partial}{\partial z_{1}}+\cdots+z_{n} \frac{\partial}{\partial z_{n}}
$$

The Euler vector field preserves the flat cone structure $Z \subset \mathbb{P} T\left(\mathbb{C}^{n}\right)$ of Definition 3.14.

The following is standard, e.g., Section 2.1 of [15].

Lemma 5.6. - Let $Z \subset \mathbb{P} V$ be a non-degenerate non-singular projective subvariety with $H^{0}(Z, T(Z))=0$. For the flat $Z$-isotrivial cone structure $Z \subset \mathbb{P} T(V)$ on $V$, the Lie algebra $\mathbf{g}$ of germs of holomorphic vector fields at $0 \in V$ preserving the cone structure has the structure of a graded Lie algebra $\mathbf{g}=\mathbf{g}_{-1}+\mathbf{g}_{0}$ with $\mathbf{g}_{-1}=V$ and $\mathbf{g}_{0}=\mathbb{C} \cdot$ E. Here $\mathbf{g}_{0}$ is the subalgebra of vector fields vanishing at 0 . 
Lemma 5.7. - Let $Z \subset \mathbb{P V}$ be a non-degenerate non-singular projective subvariety with $H^{0}(Z, T(Z))=0$. In Example 1.1, the automorphism group Aut $\left(X_{Z}\right)$ contains the vector group $\mathbb{C}^{n}$ acting with the open orbit $\mathbb{C}^{n} \cong \beta^{-1}\left(\mathbb{P}^{n} \backslash \mathbb{P}^{n-1}\right) \subset X_{Z}$. Given two connected open subsets $U_{1}, U_{2} \subset \mathbb{C}^{n}$ and a biholomorphic map $\varphi: U_{1} \rightarrow U_{2}$ preserving the locally flat VMRT-structure of $X_{Z}$, there exists $\tilde{\varphi} \in \operatorname{Aut}\left(X_{Z}\right)$ extending $\varphi$, i.e., $\varphi=\left.\tilde{\varphi}\right|_{U_{1}}$.

Proof. - The vector group $\mathbb{C}^{n}$ acts on $\mathbb{P}^{n} \backslash \mathbb{P}^{n-1} \cong \mathbb{C}^{n}$ by translations. It extends to a $\mathbb{C}^{n}$-action on $\mathbb{P}^{n}$, fixing the hyperplane $\mathbb{P}^{n-1}$. This $\mathbb{C}^{n}$-action lifts to $X_{Z}$. Fixing a base point $0 \in \beta^{-1}\left(\mathbb{P}^{n} \backslash \mathbb{P}^{n-1}\right)$, we get an open orbit $\mathbb{C}^{n} \cdot 0 \cong \beta^{-1}\left(\mathbb{P}^{n} \backslash \mathbb{P}^{n-1}\right)$.

By composing $\varphi$ with an action of the vector group in $\operatorname{Aut}\left(X_{Z}\right)$, we may assume that $0 \in U_{1}$ and $\varphi(0)=0$. Thus $\varphi$ defines an automorphism of the Lie algebra $\mathbf{g}$ in Lemma 5.6, inducing a linear transformation $\varphi^{\prime}$ of $\mathbf{g}_{-1}=V$ preserving $Z \subset \mathbb{P} V$. Then $\varphi^{\prime}$ determines an element $\tilde{\varphi}$ of $\operatorname{Aut}\left(X_{Z}\right)$, which acts on $\beta^{-1}\left(\mathbb{P}^{n} \backslash \mathbb{P}^{n-1}\right)$ as a linear transformation $\varphi^{\prime}$ on $\mathbb{C}^{n}$. Then $\varphi \circ \tilde{\varphi}^{-1}$ is a germ of biholomorphic map at $0 \in \mathbb{C}^{n}$, fixing elements of g. Since the group of affine transformations with Lie algebra $\mathbf{g}$ acts transitively on $\mathbb{C}^{n}$, this implies that $\varphi \circ \tilde{\varphi}^{-1}$ is the germ of the identity map. Thus $\varphi=\left.\tilde{\varphi}\right|_{U_{1}}$.

The next proposition is inspired by Propositions 13 in [6].

Proposition 5.8. - In the setting of Proposition 5.4, assume that $\Psi$ is not birational. Fix a general point $z \in X$. Pick $x \neq y \in X_{Z}$ with $\Psi(x)=\Psi(y)=z$. The biholomorphic map $\eta$ sending a neighborhood $U_{x}$ of $x$ to a neighborhood $U_{y}$ of $y$ induced by $\Psi$ extends to an automorphism $\tilde{\eta} \in \operatorname{Aut}\left(X_{Z}\right)$. Let $G \subset \operatorname{aut}\left(X_{Z}\right)$ be the subgroup generated by $\tilde{\eta}$ for all possible choices of $x$ and $y$. Then $G$ is a finite group in $\operatorname{Aut}\left(X_{Z}\right)$ preserving $\mathbb{C}^{n}$, acting transitively on $\Psi^{-1}(z)$.

Proof. - We may assume that $U_{x}, U_{y} \subset \beta^{-1}\left(\mathbb{P}^{n} \backslash \mathbb{P}^{n-1}\right)$. Note that $\eta: U_{x} \rightarrow U_{y}$ preserves the flat cone structure given by the VMRT-structure on $X_{Z}$, because $\Psi$ sends the VMRT-structure of $X_{Z}$ to that of $X$. This implies that $\eta$ extends to $\tilde{\eta} \in \operatorname{Aut}\left(X_{Z}\right)$ by Lemma 5.7.

Pick open subsets $W \subset X_{Z}$ and $z \in U \subset X$ such that $\Psi_{W}: W \rightarrow U$ is an unramified holomorphic map. Then $\left.\tilde{\eta}\right|_{W}$ is a covering transformation over $U$ for any choice of $x \neq y$ in the fiber $\Psi^{-1}(z)$. Thus the covering $\left.\Psi\right|_{W}$ is Galois and $G$ is the finite Galois group of $\left.\Psi\right|_{W}$.

Now we can finish the proof of Theorem 1.4. Recall that $\Psi$ in Propositions 5.4 and 5.8 has been constructed by applying Proposition 5.1 to the setting of Theorem 1.4. From Proposition 5.1 (1), the map $\Psi$ sends members of $\mathcal{K}_{Z}$ to members of $\mathcal{K}$. By Proposition 5.8, $\Psi$ factors through the quotient $X_{Z} / G$, defining a birational map $\Phi: X_{Z} / G \rightarrow X$.

\section{Proof of Theorem 1.11}

The following theorem is a direct consequence of Theorem 1.7.

$4^{\mathrm{e}}$ SÉRIE - TOME $46-2013-\mathrm{N}^{\mathrm{o}} 4$ 
Theorem 6.1. - Let $X$ be a Fano manifold of Picard number 1 different from projective space. Assume that there exists a family of minimal rational curves such that the VMRT $\mathscr{C}_{x}$ at a general point $x \in X$ is smooth and irreducible. Let $M \subset X$ be a neighborhood of a general point such that $\mathscr{C} \cap \mathbb{P} T(M)$ is a cone structure on $M$. If $v$ is a holomorphic vector field on $M$ preserving the cone structure in the sense of Definition 5.5, then $v$ extends to a global vector field $\tilde{v} \in H^{0}(X, T(X))$, i.e., $v=\left.\tilde{v}\right|_{M}$.

Theorem 6.1 has the following consequence when the VMRT-structure is locally flat.

Proposition 6.2. - Let $X$ be a Fano manifold of Picard number 1 different from projective space. Assume that there exists a family $\mathcal{K}$ of minimal rational curves such that the VMRT $\mathscr{C}_{x}$ at a general point $x \in X$ is smooth and irreducible. If $\mathscr{C}$ is locally flat at a general point $x \in X$, then the following hold.

(i) $X$ is quasi-homogeneous, i.e., the identity component $\operatorname{Aut}_{o}(X)$ of the automorphism group of $X$ has a dense open orbit $X^{o} \subset X$.

(ii) For each point $x \in X^{o}$, there exists $a \mathbb{C}^{\times}$-action on $X$ with an isolated fixed point at $x$ such that each member of $\mathcal{K}_{x}$ is the closure of an orbit of the $\mathbb{C}^{\times}$-action.

(iii) If $H^{0}\left(\mathscr{C}_{x}, T\left(\mathscr{C}_{x}\right)\right)=0$, then $X^{o}$ is affine.

Proof. - In Definition 5.5, if the cone structure is locally flat, holomorphic vector fields in a neighborhood of $x$ preserving the cone structure span the tangent space at $x$. By Theorem 6.1, these vector fields extend to vector fields on $X$. It follows that $\operatorname{Aut}_{o}(X)$ acts with a dense open orbit $X^{o}$ in $X$, proving (i).

By the local flatness, $\mathscr{C}$ is $Z$-isotrivial for some projective manifold $Z \subset \mathbb{P} V$, where $V$ is a vector space with $\operatorname{dim} V=\operatorname{dim} X$. For each point $x \in X^{o}$, the Euler vector field $E$ on $V$ from Definition 5.5 induces a local vector field $E_{x}$ in a neighborhood of $x \in X^{o}$ with an isolated zero at $x$ which preserves the locally flat cone structure $\mathscr{C}$. This vector field can be extended to a global holomorphic vector field $\tilde{E}_{x}$ on $X$ with an isolated zero at $x$, by Theorem 6.1. By integrating $\tilde{E}_{x}$, we get an analytic 1-parameter subgroup $G^{\prime} \subset \operatorname{Aut}(X)$. This analytic group $G^{\prime}$ acts on $X$ with an isolated fixed point at $x$ and the induced action on $T_{x}(X)$ is by the scalar multiplication. Let $G \subset \operatorname{Aut}(X)$ be the algebraic closure of this analytic 1-parameter subgroup $G^{\prime}$. Then $G$ is a commutative algebraic group with an isolated fixed point at $x$ with an induced representation $G \rightarrow \mathbf{G L}\left(T_{x}(X)\right)$ whose image is the diagonal multiplicative group. It follows that $G$ contains a multiplicative subgroup $G_{m} \cong \mathbb{C}^{\times}$acting as the scalar multiplication on $T_{x}(X)$. This is the desired $\mathbb{C}^{\times}$-action on $X$. To show that each member of $\mathcal{K}_{x}$ is the closure of an orbit of $G_{m}$, it suffices to show that $G_{m}$ sends each member of $\mathcal{K}_{x}$ to itself. But $G_{m}$ acts trivially on $\mathbb{P} T_{x}(X)$. Thus $G_{m}$-action deforms a member $C$ of $\mathcal{K}_{x}$ with the point $x \in C$ fixed and also the tangent direction $\mathbb{P} T_{x}(C)$ fixed. This is a contradiction to Proposition 4.1, unless the deformation is trivial, i.e., $G_{m}$ sends the curve to itself. This proves (ii).

For (iii), recall from Lemma 5.6 that if $H^{0}\left(\mathscr{C}_{x}, T\left(\mathscr{C}_{x}\right)\right)=0$, the Lie algebra of holomorphic vector fields preserving the flat cone structure $\mathcal{Z}$ on $V$ is just $V \rtimes(\mathbb{C} \cdot E)$ where $E$ is the Euler vector field. By Theorem 6.1 again, the Lie algebra of holomorphic vector fields on $X$ is isomorphic to $V \rtimes\left(\mathbb{C} \cdot \tilde{E}_{x}\right)$. It follows that $\operatorname{Aut}_{o}(X)$ contains a connected normal 
subgroup $A$ whose Lie algebra is $V$ such that $\operatorname{Aut}_{o}(X) \cong A \rtimes \mathbb{C}^{\times}$. Since $A$ is the normal subgroup, the $A$-orbit $A \cdot x$ is preserved by the $\mathbb{C}^{\times}$-action. It follows that

$$
X^{o}=\operatorname{Aut}_{o}(X) \cdot x=A \cdot x .
$$

Since $A$ is a commutative affine algebraic group with $\operatorname{dim} A=\operatorname{dim} X$ and $A \cdot x$ open in $X$, the isotropy subgroup $A_{x}$ of $A$ at $x$ is finite and $X^{o} \cong A / A_{x}$ is affine.

Proof of Theorem 1.11. - When $\operatorname{dim} X=3$, this is well-known from the classification of Fano threefolds. Thus we may assume that $\operatorname{dim} X \geq 4$. Applying Proposition 6.2, we see that $X \backslash X^{o}$ is a divisor $H$. Let $H_{1}$ be an irreducible component of $H$. All members of $\mathcal{K}_{x}$ intersect $H_{1}$ from $\operatorname{Pic}(X) \cong \mathbb{Z}$. This hypersurface $H_{1}$ is invariant under $\operatorname{Aut}_{o}(X)$, and in particular, under the $\mathbb{C}^{\times}$-action at $x$ given in Proposition 6.2 (ii). Thus the set-theoretic intersection $H_{1} \cap C$ is one point for each member $C$ of $\mathcal{K}_{x}$ because $\mathbb{C}^{\times}$-action on $\mathbb{P}^{1}$ has exactly two fixed points. On the other hand, a general member of $\mathcal{K}$ intersects $H_{1}$ transversally by the deformation theory of minimal rational curves (for example, from the splitting type of $T(X)$ in Proposition 4.1). Thus we get the intersection number $H_{1} \cdot C=1$. Since $\operatorname{deg}(\mathcal{K})=$ $C \cdot\left(-K_{X}\right)=\operatorname{dim} X$ and $\operatorname{Pic}(X) \cong \mathbb{Z}$, we see that $-K_{X}=(\operatorname{dim} X) \cdot H_{1}$ in $\operatorname{Pic}(X)$. Thus $X$ is biregular to the hyperquadric by Kobayashi-Ochiai criterion [10].

\section{REFERENCES}

[1] C. Araujo, Rational curves of minimal degree and characterizations of projective spaces, Math. Ann. 335 (2006), 937-951.

[2] K. Cho, Y. Miyaoka, N. I. Shepherd-Barron, Characterizations of projective space and applications to complex symplectic manifolds, in Higher dimensional birational geometry (Kyoto, 1997), Adv. Stud. Pure Math. 35, Math. Soc. Japan, 2002, $1-88$.

[3] J. Hong, J.-M. Hwang, Characterization of the rational homogeneous space associated to a long simple root by its variety of minimal rational tangents, in Algebraic geometry in East Asia-Hanoi 2005, Adv. Stud. Pure Math. 50, Math. Soc. Japan, 2008, 217-236.

[4] J.-M. Hwang, Geometry of minimal rational curves on Fano manifolds, in School on Vanishing Theorems and Effective Results in Algebraic Geometry (Trieste, 2000), ICTP Lect. Notes 6, Abdus Salam Int. Cent. Theoret. Phys., Trieste, 2001, 335-393.

[5] J.-M. HwaNG, Equivalence problem for minimal rational curves with isotrivial varieties of minimal rational tangents, Ann. Sci. Éc. Norm. Supér. 43 (2010), 607-620.

[6] J.-M. Hwang, N. Mok, Holomorphic maps from rational homogeneous spaces of Picard number 1 onto projective manifolds, Invent. Math. 136 (1999), 209-231.

[7] J.-M. Hwang, N. Mok, Cartan-Fubini type extension of holomorphic maps for Fano manifolds of Picard number 1, J. Math. Pures Appl. 80 (2001), 563-575.

[8] J.-M. Hwang, N. Mok, Birationality of the tangent map for minimal rational curves, Asian J. Math. 8 (2004), 51-63.

[9] S. Kebenus, Families of singular rational curves, J. Algebraic Geom. 11 (2002), 245256.

4 e SÉRIE - TOME $46-2013-$ No $^{\circ}$ 
[10] S. Kobayashi, T. OchiaI, Characterizations of complex projective spaces and hyperquadrics, J. Math. Kyoto Univ. 13 (1973), 31-47.

[11] J. Kollár, Rational curves on algebraic varieties, Ergebn. Math. Grenzg. 32, Springer, 1996.

[12] Y. Miyaoka, Numerical characterisations of hyperquadrics, in Complex analysis in several variables-Memorial Conference of Kiyoshi Oka's Centennial Birthday, Adv. Stud. Pure Math. 42, Math. Soc. Japan, 2004, 209-235.

[13] N. Mok, Recognizing certain rational homogeneous manifolds of Picard number 1 from their varieties of minimal rational tangents, in Third International Congress of Chinese Mathematicians. Parts 1, 2, AMS/IP Stud. Adv. Math. 42, Amer. Math. Soc., 2008, 41-61.

[14] C. Okonek, M. Schneider, H. Spindler, Vector bundles on complex projective spaces, Progress in Math. 3, Birkhäuser, 1980.

[15] K. Yamaguchi, Differential systems associated with simple graded Lie algebras, in Progress in differential geometry, Adv. Stud. Pure Math. 22, Math. Soc. Japan, 1993, 413-494.

\author{
Jun-Muk Hwang \\ Korea Institute for Advanced Study \\ Hoegiro 87 \\ Seoul, 130-722, Korea \\ E-mail: jmhwang@kias.re.kr
}

